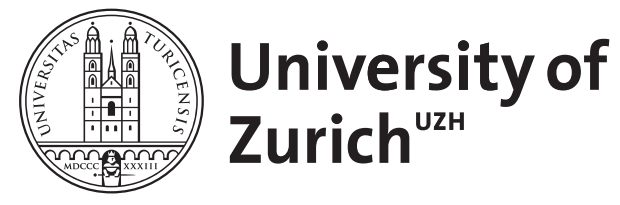

Zurich Open Repository and Archive

University of Zurich

University Library

Strickhofstrasse 39

CH-8057 Zurich

www.zora.uzh.ch

Year: 1996

Proclus politisé : La réception politique de Proclus au moyen âge tardif

Cheneval, Francis

DOI: https://doi.org/10.1515/agph.1996.78.1.11

Posted at the Zurich Open Repository and Archive, University of Zurich

ZORA URL: https://doi.org/10.5167/uzh-52536

Journal Article

Published Version

Originally published at:

Cheneval, Francis (1996). Proclus politisé : La réception politique de Proclus au moyen âge tardif. Archiv für Geschichte der Philosophie, 78(1):11-26.

DOI: https://doi.org/10.1515/agph.1996.78.1.11 


\title{
Proclus politisé: La réception politique de Proclus au moyen âge tardif
}

\author{
de Francis Cheneval (Zürich)
}

\section{Introduction}

Wenn ich alle transszendentale Ideen, deren Inbegriff die eigentliche Aufgabe der natürlichen reinen Vernunft ausmacht, welche sie nöthigt, die bloße Naturbetrachtung zu verlassen und über alle mögliche Erfahrung hinauszugehen und in dieser Bestrebung das Ding (es sei Wissen oder Vernünfteln), was Metaphysik heißt, zu Stande zu bringen, zusammennehme: so glaube ich gewahr zu werden, daß diese Naturanlage dahin abgezielt sei, unseren Begriff von den Fesseln der Erfahrung und den Schranken der bloßen Naturbetrachtung so weit loszumachen, $\mathrm{da} ß$ er wenigstens ein Feld vor sich eröffnet sehe, was blos Gegenstände für den reinen Verstand enthält, die keine Sinnlichkeit erreichen kann, zwar nicht in der Absicht, um uns mit diesen speculativ zu beschäftigen (weil wir keinen Boden finden, worauf wir Fuß fassen können), sondern damit praktische Principien, die, ohne einen solchen Raum für ihre nothwendige Erwartung und Hoffnung vor sich zu finden, sich nicht zu der Allgemeinheit ausbreiten könnten, deren die Vernunft in moralischer Absicht unumgänglich bedarf. ${ }^{1}$

J'entreprends mon exposé avec cette citation de Kant afin de tenter de justifier le fait que la métaphysique en tant que telle est presque absente de mon propos, bien que l'objet de cet article, Proclus, m'obligerait en principe à l'évoquer bien davantage. Je cite le texte de Kant parce qu'il thématise le problème de la relation entre métaphysique et philosophie pratique d'une façon qui me semble paradigmatique au moins pour ce que je tenterai de montrer dans cet article. Le texte de Kant suggère que la philosophie pratique doit beaucoup à la métaphysique; elle est sa condition de possibilité. Sans la métaphysique, la philosophie pratique n'atteindrait pas le degré d'universalité dont elle a besoin pour arriver à ses preuves. D'autre part, la métaphysique reste nécessairement liée à l'empirie et à sa fin pratique; outre sa finalisation

1 Immanuel Kant, Prolegomena zu einer jeden künftigen Metaphysik, die als Wissenschaft wird auftreten können, §60; AA, IV, $362 \mathrm{f}$.

Archiv f. Gesch. d. Philosophie 78. Bd., S. 11-26

(C) Walter de Gruyter 1996

ISSN 0003-9101 
pratique, elle ne possède aucun statut indépendant. Conçue comme spéculation pure, elle ne trouverait pas de sol sous son pied. Nous pouvons accepter ou rejeter cette conception kantienne de la métaphysique, elle peut même nous paraître circulaire. Mais quoi qu'il en soit, d'un point de vue historique, on peut démontrer que la structure de la démarche, consistant en une finalisation et instrumentalisation pratique et politique de la métaphysique, a été suivie, consciemment ou inconsciemment, bien avant Kant et sans la réflexion théorique avec laquelle le philosophe de Königsberg avait légitimé sa démarche. Afin de parvenir à mener à bien son discours, la philosophie pratique et politique a souvent instrumentalisé le système d'argumentation métaphysique dans son interêt, avec la conséquence que la métaphysique n'a guère pu demeurer affranchie de son environnement pratique et politique. C'est de ce problème que traitera mon exposé. Plutôt que de présenter la réception de la métaphysique proclusienne au moyen âge en tant que métaphysique pure, je proposerai de considérer le rôle de la métaphysique du point de vue de sa finalisation et instrumentalisation pratique, me limitant en ceci au cas exemplaire de la réception politique de Proclus au moyen âge tardif. Je tenterai de démontrer que, en matière de réduction de la métaphysique à la politique, la réception de la pensée de Proclus ne se démarque pas de la règle générale. On peut démontrer que certains éléments textuels de Proclus ont été mis au service d'un discours de légitimation politique. Cela ne veut pas dire que les auteurs dont je vais proposer la lecture dans la suite partagent avec Kant la conception systématique de la métaphysique et de son lieu dans le système philosophique. Mais le texte de Kant peut nous servir comme cadre méthodique dans lequel se joue l'instrumentalisation politique de la métaphysique dont quelques auteurs nous feront témoignage. C'est d'une façon formelle et d'un point de vue fonctionnaliste que leur démarche est identique à celle de Kant: ils finalisent et instrumentalisent la métaphysique pour arriver à une preuve de nature politique. Avec cela ils donnent à la métaphysique un rôle important que nous ignorons si nous nous limitons à l'analyse intrinsèque ou immanente des textes métaphysiques. Le but méthodique de mon exposé serait donc de montrer l'importance et l'utilité de l'approche fonctionnaliste aux textes de l'histoire de la philosophie.

La thèse d'un Proclus politisé au moyen âge peut paraître simple, mais je dois affirmer que la lecture politique de Proclus a été jusqu'à présent négligée par l'historiographie. Mon exposé se voudrait donc, en ce sens, comme un modeste appendice aux grands travaux sur Proclus au moyen âge. Parmi les plus récents de ceux-ci, il faut mentionner 
Denken des Einen de Werner Beierwaltes, un ouvrage dans lequel l'auteur expose la Wirkungsgeschichte de l'idée néoplatonicienne de l'un au moyen âge. ${ }^{2}$ Aux résultats multiples livrés par ce travail, j'aimerais ajouter la dimension politique de la réception médiévale de la philosophie de Proclus, une question fort négligée dans le récent On Proclus and his influence in medieval philosophy, édité par E. P. Bos ${ }^{3}$, de même que dans les actes du colloque "Proclus et son influence», qui a eu lieu à Neuchâtel en $1985 .^{4}$ Ce dernier ouvrage, quoi qu'il en soit, présente une étude de Loris Sturlese qui n'est pas sans importance pour notre propos. Intitulé «Il dibattito sul Proclo Latino», ce texte propose l'analyse de nombreuses citations de Proclus au cours de l'époque allant de 1260 à 1350 . Les conclusions générales très bien fondées auxquelles arrive l'auteur pourront servir de cadre général à notre intention de comprendre la réception politique de Proclus, et j'aimerais les présenter ici. Loris Sturlese arrive tout d'abord à la conclusion que la réception de Proclus, à l'exception du cas particulier de l'école des Dominicains de Cologne, est très vite devenue fort éclectique, et s'est limitée à quelques éléments isolés de sa philosophie. Il s'agissait, pour citer le savant italien, d'une "stilizzazione delle tesi dell'Elementatio theologica a pochi luoghi comuni $»{ }^{5}$ Sturlese démontre en second lieu que, sous l'influence d'Albert le Grand, nombre d'auteurs ont tenté d'harmoniser la métaphysique aristotélicienne et la métaphysique proclusienne. ${ }^{6}$ C'est en arrière-plan à ces thèses générales que j'aimerais présenter ici quelques textes qui permettront d'étayer ma thèse, selon laquelle on a pu alors assister à une remarquable mise en fonction politique de certains éléments textuels proclusiens. Bien que nonexhaustif, nous espérons que ce choix de textes pourra permettre de distinguer les différentes politisations de Proclus, de même qu'il pourra servir à illustrer quelques aspects de la relation entre les diverses réceptions politiques des métaphysiques aristotélicienne et proclusienne.

2 W. Beierwaltes, Denken des Einen. Studien zur neuplatonischen Philosophie und ihrer Wirkungsgeschichte, Frankfurt 1985.

3 E. P. Bos/P. A. Meijer (eds.), Proclus and his influence in medieval philosophy (Philosophia antiqua: a series of monographs on ancient philosophy, 53), Leiden 1992.

4 Proclus et son influence, Actes du colloque de Neuchâtel juin 1985, édités par G. Boss et G. Seel, avec une introduction de F. Brunner, Zürich 1987.

5 L. Sturlese, «Il dibattito sul Proclo latino nel medioevo fra l'università di Parigi e lo studium di Colonia », dans: G. Boss/G. Seel (eds.), Proclus et son influence, 261.

6 Cf. Ibidem, 269. 


\section{Les textes}

\section{Proclus et l'ecclésiologie}

Le premier exemple d'une politisation de Proclus que nous allons présenter ici survient quand une proposition des Élements de théologie est mise au service du discours ecclésiologique dans le Tractatus cuius titulus reprobatio errorum de Guillaume de Crémone. Ce traité faisait partie de l'arsenal de mesures assemblé par l'Église officielle dans sa lutte contre le Défenseur de la paix de Marsile de Padoue, publié en 1324. ${ }^{7}$ Une commission établie par le pape Jean XXII avait envoyé à plusieurs canonistes une liste de cinq ou six erreurs contenues dans l'ouvrage de Marsile, avec pour tâche d'en rédiger une réfutation. Guillaume de Crémone accomplit brillamment cette mission avec son Reprobatio errorum, et livrait à Jean XXII le fondement théorique de la bulle Licet iuxta doctrinam, par laquelle le pape condamnait le Défenseur de la paix le 23 octobre $1327 .{ }^{8}$ Dans ce passage, qui se trouve dans la partie du tractatus où est traité le pouvoir du pape dans l'Église et où les thèses conciliaristes de Marsile sont réfutées, Guillaume fait appel à Proclus pour démontrer une thèse de nature politique. Nous assistons donc à la mise en fonction de Proclus dans un discours ecclésiologique.

7 Marsilius von Padua, Defensor Pacis, herausgegeben von Richard Scholz (Fontes Iuris Germanici Antiqui in usum scholarium ex Monumentis Germaniae Historicis separatim editi), Hannover 1932. Pour une bibliographie cf. J. Miethke, «Literatur über Marsilius von Padua (1958-1992)», dans: Bulletin de Philosophie Médiévale 35 (1993) 150-165.

8 "Errores, quos dicunt et asserunt quidam magistri, ex parte sanctissimi patris et domini nostri, domini Joannis papae vigesimi secundi mihi missi, ut super eis scriberem, quod mihi videretur, sunt isti infrascripti per hunc modum. » (Guillelmus Cremonensis O.S. A., Tractatus cuius titulus Reprobatio errorum. Ed. D. Mac Fhionnbhairr. (Corpus Scriptorum Augustinianorum, IV) Rome 1977, 3). La littérature sur Guillaume de Crémone n'est pas abondante. Cf. R. Scholz, Unbekannte kirchenpolitische Streitschriften aus der Zeit Ludwigs des Bayern (1327-1354), 2 vol. Rome 1913, 13-22; U. Mariani, «Un avversario di Marsilio da Padova: Guglielmo Amidani da Cremona ", dans: Giornale Dantesco 30 (1927) 73-88; Idem, Chiesa e Stato nei Teologi Agostiniani del secolo XIV, (Uomini e dottrine, 5), Rome 1957, 103-111, 203-214; W. Kölmel, Regimen Christianum. Weg und Ergebnisse des Gewaltenverhältnisses, 8. -14. Jahrhundert, Berlin 1970, 439 f.; D. Del Prete, «La confutazione del Defensor pacis di Marsilio da Padova: de Siberto de Beek, Guglielmo Amidani, Pietro da Lutra », dans: Annali del Dipartimento di Scienze Storiche e Sociali. Univ. degli studi di Lecce 1 (1982) 213-283. 
Secundum Proclum omnis multitudo bene ordinata reducitur ad unum. Sed Ecclesia Christi est quaedam multitudo et bene ordinata. [...] Ergo oportet, quod habeat aliquod unum, ad quod reducatur. ${ }^{9}$

On a donc recours à la proposition 21 des Éléments de théologie de Proclus ${ }^{10}$ pour légitimer la monarchie papale, et contrer la thèse de Marsile selon laquelle le pouvoir dans l'Église demeure entre les mains de la communauté des fidèles. La proposition est présentée comme proposition universelle, comme maior dans un syllogisme du troisième mode de la première figure, ${ }^{11}$ et sa conclusion consiste à affirmer la nécessité du gouvernement monarchique dans l'Église. Au raisonnement pratique de Guillaume de Crémone, la proposition de Proclus confère l'indispensable attribut d'universalité.

Notons que ‘Église〉 est compris par Guillaume dans un sens purement philosophique, comme structurellement identique à toute multitude ordonnée, et c'est Proclus, le philosophe païen, qui lui livre l'argument justifiant le principe selon lequel cette multitude ne doit être dirigée que par un seul homme. La réduction à l'un n'est pas limitée à son contexte métaphysique pur, mais mise au service de la légitimation du pouvoir monarchique du pape au sein de l'Église; elle sert de principe général pour combattre la théorie démocratique de Marsile de Padoue, et pour légitimer une forme de domination de type despotique.

\section{Proclus et le pouvoir universel du pape}

La mise en fonction de la proposition proclusienne de la réduction à l'un de toute multitude ne se limita pas à légitimer le pouvoir papal au sein de l'Église, mais fut également appelée à soutenir la prétention papale au pouvoir absolu en matières temporelles. C'est dans le Tractatus de potestate summi pontificis de Guido Vernani de Rimini que l'on retrouve le meilleur exemple de cette utilisation. ${ }^{12}$ Composé vers 1327 ,

9 Tractatus cuius titulus, Q. 3, 1: Ed. Mac Fhionnbhairr, 68.

10 Cf. Proclus, Elementatio Theologica translata a Guillelmo de Morbeca, herausgegeben von H. Boese, Louvain 1987, $14 \mathrm{f}$.

11 " tertius constat ex universali affirmativa et particulari affirmativa particularem affirmativam concludentibus" (Petrus Hispanus, Tractatus called afterwards Summule Logicales, ed. L. M. De Rijk, Assen 1972, 46).

12 Pour Vernani cf. Th. Käppeli, "Der Dantegegner Guido Vernani von Rimini », dans: Quellen und Forschungen aus italienischen Archiven und Bibliotheken 28 (1937-38) 107-146; Idem, Scriptores Ordinis Predicatorum medii aevi, II, Rome 1970-, 76 f.; N. Matteini, Il più antico oppositore di Dante: Guido Vernani da 
ce traité, dont l'édition critique vient de paraître, est important sous plusieurs aspects. Il s'inscrit clairement dans la lignée des traités dits De potestate pape, à la différence qu'ici, le commentateur d'Aristote qu'était Vernani cite de longs passages de ses commentaires de l'Ethique et de la Politique dans le but de conférer une légitimation philosophique au pouvoir absolu du pape. Nous retrouvons donc un Aristote défenseur du pouvoir papal, de même qu'un Proclus légitimant l'universalisation de la communauté politique afin de la soumettre à ce même pouvoir. Car la proposition 21, sur la réduction à l'un, apparaît dans le chapitre 3 du traité de Vernani, au sein d'une discussion sur la prédominance naturelle du pouvoir spirituel au niveau de la communauté politique:

Capitulum Tertium. Quod potestas spiritualis debet principari in comunitate civitatis et rei publice.

(1) Post comunitatem domesticam sequitur comunitas civitatis, que secundum Aristotelem primo Politicorum est etiam comunitas naturalis ${ }^{13}$, eo quod ad talem comunicationem comuniter homines naturaliter inclinantur. Hec autem comunitas divinitus et naturaliter instituta debet esse per se sufficiens ad vitam et quantum ad ea, que sunt necessaria corpori, et quantum ad ea, que sunt necessaria bone vite. Non enim est civitas instituta solum propter vivere, sed propter beate vivere, ut dicit Aristoteles primo Politicorum ${ }^{14}$.

(2) Oportet autem secundum sententiam ante dictam, cum civitas sit ex pluribus constituta, quod in ipsa sit principans et subiectum, sive regens et rectum, sive imperans et quod debet obedire precepto.

(3) Est autem considerandum, quod sicut propagatione nature multiplicatis personis ex una domo exeunt plures domus, et multe domus constituunt unum vicum, et multi vici constituunt civitatem, et multe civitates constituunt unam provinciam, et multe provincie constituunt unum regnum, ita multa regna ad

Rimini, Padoue 1958, 7-18; J. Dunbabin, «Guido Vernani of Rimini's Commentary on Aristotle's Politics», dans: Traditio 44 (1988) 373 f.; A. Meozzi, «I trattati politici di Guido Vernani e Dante Alighieri», dans: Giornale Dantesco 30 (1927) 18-30; P. G. Ricci, "Un difficile e importante passo della Monarchia », dans: Studi danteschi 42 (1965) 361-368; B. Nardi, « Di un'aspra critica di fra Guido Vernani a Dante », dans: Saggi e note di critica dantesca, Milan 1966, 377-385; A. Vallone, Antidantismo politico nel XIV secolo, Naples 1973, 60-70; Ch. Lohr, "Medieval Latin Aristotle Commentaries, Authors G-I», dans: Traditio 24 (1968) 191 f.; J. De Raedemaeker, "Informations concernant quelques commentaires du De Anima ", dans: Bulletin de Philosophie Médiévale 8-9 (1966-67) 102-104; Ch. Flüeler, (Rezeption und Interpretation der Aristotelischen Politica, I, Amsterdam 1992, 65 f.) discute des thèses dans le commentaire de Vernani de la Politique sur la servitus; F. Chevenal, Die Rezeption der Monarchia Dantes bis zur Editio Principes im Jahre 1559, München 1995, 117-150.

$131253 \mathrm{a} 2$.

$141252 \mathrm{~b} 30$. 
unum imperium reducuntur. Omnis enim multitudo reducitur ad unitatem, ut dicit Proclus ${ }^{15}$. Et sic sequitur, quod imperium est unum solum, cui omnes secundum legem nature et secundum deum, qui est institutor nature, debent subesse et eius iustis legibus et preceptis obedire.

(4) Iuste autem leges esse dicuntur, que a naturali ratione procedunt, ut patet per Aristotelem, 5. Ethicorum ${ }^{16}$, ubi etiam dicit, quod non sinimus principari hominem sed rationem. Ergo patet, quod in isto tertio perfectiori rei publice principatu spiritualis potestas debet principaliter dominari, que principaliter est in summo pontifice divinitus constituta. ${ }^{17}$

On voit que la citation de Proclus est combinée à la théorie aristotélicienne de la domination naturelle dans toute multitude. Par rapport au texte de Guillaume de Crémone, la proposition proclusienne a changé de fonction dans l'argumentation. La théorie de la dominance de l'un dans toute multitude est livrée par Aristote, dans le paragraphe $2 \mathrm{du}$ texte, et non pas par Proclus, comme c'était le cas chez Guillaume. Dans le texte de Vernani, la réduction à l'un de Proclus sert à justifier la réduction par étapes de toute communauté à la communauté universelle (3). Proclus légitime ici l'universalisme politique. Dans le paragraphe (4) Vernani associe la preuve aristotélicienne de la domination naturelle de toute communauté par le pouvoir spirituel à la réduction proclusienne de toute communauté à la communauté universelle. Il en tire la conclusion d'un pouvoir universel attaché à la personne spirituelle du pape. La réduction à l'un, en définitive, autorise la domination universelle de l'un.

On retrouve une variante de cet amalgame entre Aristote et Proclus, toujours en vue de légitimer le pouvoir universel du pape, dans la Glossa ad Extravagantes (1.V, tit. 9, De poenit. et remiss., c.1). La proposition 21 ne justifie pas ici, comme c'était le cas chez Vernani, la réduction de toute communauté à la communauté universelle; mais l'argument de la réduction à l'un est parallèle à celui de Mét. X, 1 d'Aristote, selon lequel il y a dans tout genre un premier par lequel les autres sont mesurés. Le regroupement d'éléments isolés de la métaphysique d'Aristote et de celle de Proclus sert de la sorte de fondement théorique de la plenitudo potestatis du pape.

Huic autem veritati concordat ratio recta. Nam secundum Proclum, omnis multitudo reducitur ad unitatem. Et secundum Philosophum, in unoquoque genere est reperire unum primum et supremum, quod sit mensura et regula omnium aliorum

15 Elementatio theologica, prop. 21: Ed. Boese, $14 \mathrm{f}$.

$161134 \mathrm{a} 35$.

17. Tractatus de potestate summi pontificis, 3: Ed. Chevenal, Die Rezeption der Monarchia, $426 \mathrm{f}$. 
in illo genere contentorum [...]. Oportet igitur quod multitudo hominum reducatur ad unum, et in genere hominum sit reperire unum hominem primum, qui sit supremum in illo genere, qui sit mensura et regula omnium aliorum et hominum: huiusmodi autem est romanus pontifex, qui est inter homines supremus, existens mensura et regula directiva omnium aliorum, cui plene omnes catholici sunt subiecti [...] tunc omnes nationes orbis terrarum huic unico capiti subijcentur et obedientur. ${ }^{18}$

L'auteur de la Glose n'établit aucune distinction entre la réduction à l'un de la Métaphysique d'Aristote et la réduction à l'un de la métaphysique proclusienne. Il les cite de façon complémentaire, et en déduit la réduction de toute communauté humaine au pape.

J'ai signalé plus haut que cet exposé n'offrirait qu'un choix de textes restreint, mais suffisamment développé pour permettre une démonstration adéquate de la thèse d'un Proclus politique au moyen âge tardif. Afin de démontrer que la réception politique de Proclus alla aussi au delà de la proposition 21 (ou 5) des Éléments de théologie, nous pourrions évoquer deux autres textes qui assemblent une argumentation politique sur la base du Liber de causis ou des Éléments de théologie. Afin de démontrer que tout pouvoir vient de Dieu ex parte motus, la Determinatio compendiosa, attribuée à Ptolémée de Lucques, fait mention, de façon très générale, à la fois aux Éléments de théologie et au Liber de causis comme autorités ayant conçu l'ordre graduel de l'être selon la noblesse des choses. ${ }^{19}$ La Physique et la Métaphysique d'Aristote, les Élements de théologie et le Liber de causis, ainsi qu'un passage du De Trinitate d'Augustin, sont interprétés en parfaite harmonie les uns avec les autres, afin de légitimer l'origine divine du pouvoir politique:

Quod dominium hominum est a deo, ratio sic sumitur ex parte motus:

Secunda vero ratio, quod omne dominium sit a Deo, sumitur in comparatione ad motum. Probat enim phylosophus in VIII. de Physico auditu, quod in moventibus et motis non est in infinitum abire, sed est venire ad aliquod movens, quod

18 Glossa ad Extravagantes communes, lib. V, tit. IX, Basel: Froben 1511, fol. 35v; Basel UB: N.n. V. 12, No. 4.

19 Pour la Determinatio compendiosa qui aurait mérité qu'on la mentionne dans la Cambridge History of Medieval Political Thought cf. H. Grauert, «Aus der kirchlichen Traktatenliteratur des 14. Jahrhunderts", dans: Historisches Jahrbuch 29 (1908) 497-536; J. Miethke, «Die Rolle der Bettelorden an der Wende zum 14. Jahrhundert", dans: K. Elm (Hrsg.), Stellung und Wirksamkeit der Bettelorden in der städtischen Gesellschaft (Berliner Hist. Studien, 3, II), Berlin 1981, $124 \mathrm{ff}$; Idem, «Die Traktate «De potestate pape» », dans: Les genres littéraires dans les sources théologiques et philosophiques médiévales - Actes du Colloque intern. de Louvain-la-Neuve 25-27 mai 1981, Louvain 1982, 195. 
non movetur, et hoc est Deus. [...] Hanc eandem rationem tradit beatus Augustinus in III. de Trinitate, ubi ordinem motoris et mobilis ponens incipit ab infimo corpore, puta terra, per subtiliora et virtusiora dicit grossiora moveri, gradatim ascendens secundum ordinem elementorum usque ad supremum corpus et ulterius ad spiritus rationales, per quos dicit corpora superiora moveri, spiritus vero rationales inferiores per superiores, sed omnes spiritus rationales creatos per spiritum rationalem increatum, qui est Deus, in quo consistit tota ratio motus sicut in principali movente immobili, in quo est omne ens, motus et vita [...]. Et hunc ordinem Plato posuit secundum nobilitatem et gradum entis, ut patet ex propositionibus Proculi et ex libro de Causis. [...]. Quam conclusionem etiam a phylosopho habere possumus in XII. Prime phylosophie, ubi primam causam, que Deus est, comparat ad totum universum sicut ducem ad exercitum. ${ }^{20}$

Il ne s'agit pas ici de la réception partielle et politisée d'une proposition isolée des Éléments de théologie, mais de l'idée d'une gradation de l'être selon l'ordre de noblesse ontologique que l'on attribue à la fois aux Éléments de théologie, au De Trinitate d'Augustin, au livre 12 de la Métaphysique d'Aristote et au Liber de causis, et ce, dans le but de justifier la continuité et le lien de causalité entre pouvoir divin et pouvoir temporel. La Determinatio compendiosa en tire la conclusion, au chapitre 25, que le pouvoir temporel du pape, vicaire du Christ, est d'origine divine, et que, par conséquent, il est dépositaire de la plénitude du pouvoir.

Gilles de Rome, un des plus importants défenseur du pouvoir universel du pape, en se référant au Liber de causis dans son De ecclesiastica potestate nous livre un autre exemple d'une réception politique de Proclus. Cela démontre que la réception politique de Proclus ne se limitait pas à la proposition 21 ou 5 des Éléments de théologie:

Sic et Deus est quoddam mare perfeccionum, in quo sunt perfecciones omnes, iuxta illud quod habetur in quinto Metaphysice, quod est quoddam perfectum, in quo congregantur perfecciones omnium generum: sic est quoddam mare potenciarum et virtutum, quia est in eo omnis potencia et omnis virtus. Unde et omnes alie virtutes sunt pendentes per virtutem primam et sunt derivate a virtute prima. Ideo dicitur in decima sexta proposicione De Causis, quod omnes virtutes sunt pendentes per infinitum primum, quod est virtus virtutem. Sunt ergo diversa genera virtutem et potenciarum, quia alique sunt intellectuales, alique sensibiles, alique celestes, alique terrene; sed omnia hec non a pluribus fontibus nec a pluribus primis principiis, sed $a b$ uno fonte et $a b$ uno primo principio derivantur. Hiis itaque prelibatis adaptemus hoc ad propositum et dicamus, quod sicut in gubernacione tocius mundi unus est fons, unus est Deus, in quo est omnis potencia, a quo omnes alie potencie derivantur, et in quo omnes potencie reducuntur,

20 Ed. M. Krammer, Hannover/Leipzig 1909, 39 ff. 
sic et in gubernacione hominum et in tota ecclesia militante oportet, quod unus sit fons, unum sit caput, in quo sit plenitudo potestatis, in quo sit omnis potencia [...] apud quem sit uterque gladius, quia aliter non esset in eo omnis potencia.

[...] Sicut ergo censendum est de Deo, prout secundum legem communem gubernat totum mundum, sic eciam censendum est de vicario Dei. ${ }^{21}$

La proposition 16 du Liber de causis sert à Gilles de Rome pour prouver le monothéisme afin d'en dériver la monocratie papale. La gubernacio de Dieu dans le monde corresponde à la gubernacio de son vicaire. La formule (vicarius christi), ${ }^{22}$ plus tard sévèrement critiquée par Dante, ${ }^{23}$ permet d'attribuer au pape les attributs de pouvoir de Dieu. Monothéisme, monisme cosmologique et monocratie politique forment ainsi dans le système de Gilles une unité qui donne à la métaphysique de fortes implications politiques. Une fois de plus c'est la métaphysique de l'un de Proclus qui légitime la domination de l'un, à savoir la domination du pape dans le monde entier.

\section{Proclus et la théorie de la domination naturelle}

Les défenseurs du pouvoir monarchique des papes ne furent pas les seuls à faire usage de la proposition de Proclus. La réception politique de celui-ci, d'ailleurs, ne fut pas l'apanage exclusif des propagateurs de théories hiérocratiques. On retrouve ainsi une mise en fonction politique de Proclus dans un texte philosophique universitaire, à savoir dans les Questiones supra libros politicorum de Pierre d'Auvergne. ${ }^{24}$ Dans la question 12 du premier livre, Pierre aborde la question de la domination et de l'esclavage naturel dans toute communauté. Deux arguments sont mis en avant pour soutenir le principe d'une domination naturelle, et, contre toute attente, l'un d'eux consiste en la proposition 21 des Éléments de théologie de Proclus:

Utrum ubicunque est coniunctio aliquorum in unum, sit unum principans natura et aliud servum et subiectum. Arguitur quod non, quia [...]. Contra arguitur: Omnis multitudo necessario reducitur ad unum, quia omnis multitudo secunda est ab uno, ut dicit Proclus. Item. Primum in unoquoque 〈genere〉 est causa omnium

21 Gilles de Rome, De ecclesiastica potestate, III, 2: Ed. R. Scholz, Weimar 1929, $151 \mathrm{f}$.

22 Pour l'histoire de ce titre politique cf. M. Maccarrone, Vicarius Christi: Storia del titolo papale, Rome 1952.

23 Cf. Mon. III, vi: Ed. Ricci, Milan 1965, 242-245.

24 Pour ce texte cf. C. Flüeler, Rezeption und Interpretation, I, 86-131. 
aliorum, sed hoc non potest fieri, nisi aliquid est princeps et subiectum. Ergo etc. Dicendum, quod in omnibus ex quibus coniunctis fit unum per se, non secundum accidens, in illis alquid 〈sic〉 est princeps et aliquid subiectum. ${ }^{25}$

Pierre d'Auvergne insère des parties des propositions $5^{26}$ et 21 des Éléments de théologie dans une argumentation en faveur de la domination naturelle dans toute multitude non substantielle. Procédant comme l'avait fait la très légaliste Glose, le philosophe Pierre d'Auvergne combine et harmonise la réduction à l'un de Proclus avec l'argument aristotélicien de Mét. X, c.1: 1052b-1053a20: dans tout genre il se trouve un premier par lequel les autres sont mesurés, et ce principe est constitutif du genre. Ainsi réunies, les formules de Proclus et d'Aristote jettent les bases d'une justification de la domination naturelle d'un premier dans toute communauté composée. Au delà d'une légitimation du pouvoir papal, Pierre d'Auvergne fait usage de la proposition 21 des Éléments de théologie pour échafauder une théorie générale de la domination de type despotique, applicable à toute communauté humaine.

\section{Observations et conclusions}

\section{Une réception éclectique}

Les textes que nous venons d'examiner présentent une similitude qui n'aura échappé à personne: ils font presque tous appel à la même proposition des Éléments de théologie de Proclus, à savoir la proposition 21: "Omnis ordinis multitudo ad unam reducitur unitatem " 27 . Il s'agit là, à n'en pas douter, d'une réception extrêmement fragmentaire des Éléments de théologie: On a isolé une proposition afin de la mettre au service d'une argumentation politique. Les auteurs examinés se rendent coupables de ce que Chalcide reprochait déjà à Aristote dans son commentaire du Timée: ils isolent à leur gré une phrase de son contexte, en négligeant le reste du système: «suo quodam more pleni perfectique dogmatis electo quod visum sit cetera fastidiosa incuria neglegit ${ }^{28}$.

25 Petrus de Alvernia, Questiones in libros politicorum, I, 12: Ed. C. Flüeler, Amsterdam 1989, 186.

26 Ed. H. Boese, Louvain 1987, $4 \mathrm{f}$.

27 Ibid., $14 \mathrm{f}$.

28 Chalcidius, In Tim., 250: Ed. J. H. Waszink (Plato Latinus, IV, ed. R. Klibansky), London/Leiden 1962. 
On pourrait reprocher aux textes ici discutés qu'ils se distinguent de la vraie philosophie par un éclectisme qui est causé par l'utilisation propagandiste de textes métaphysiques. Mais à la lumière des conclusions de Loris Sturlese sur la réception latine de Proclus, on doit constater que l'éclectisme observé chez les auteurs politiques n'est pas la manifestation d'une philosophie défigurée par des intérêts de politique et de propagande, mais qu'il est partie prenante d'une deuxième phase de la réception de Proclus au moyen âge tardif. Les textes qui font un usage politique de Proclus ne font que confirmer la nature éclectique de sa réception, la «stilizzazione a pochi luoghi comuni».

\section{La fonction des proposition 21 ou 5 de Proclus dans le discours politique}

Appliquée comme axiome, comme principe universel, la proposition 21 présente le caractère d'universalité qui permet à la philosophie pratique d'étayer ses preuves. Il est intéressant de reconnaître dans la réception de Proclus une transformation de la fonction logique de cette proposition. Ainsi que l'a démontré James Lowry ${ }^{29}$, les propositions des Éléments de théologie sont présentées comme des conclusions aux démonstrations leur succédant. C'est également le cas de la proposition 21. Dans la mise en fonction politique présentée ici, la proposition sert d'axiome, de maior, au sein d'une démonstration.

Bien que ce type de métaphysique et de preuve étaient absents du propos de Kant, quand il songeait à une nécessaire finalisation de la métaphysique à la politique, il me semble que la structure de l'argumentation kantienne est identique à celle proposée dans les textes cités ici, puisque la philosophie pratique se base sur des principes universels, dits métaphysiques, pour transmettre à ses résultats particuliers le plus haut degré d'universalité.

3. Les différents types de mise en fonction politique de Proclus

Il nous faut signaler le fait que, en matière de politisation de Proclus, les passages que nous venons d'examiner présentent entre-eux certaines variations et subtilités bien qu'à une exception près ils traitent tous de la même phrase. La mise en fonction de la proposition 21 présentée par

29 The Logical principles of Proclus' Stoicheiosis Theologike as systematic ground of the cosmos, Amsterdam 1980, 37. 
Guillaume de Crémone se veut favorable au pouvoir monarchique du pape dans l'Église. Tout autres sont les textes de Guido Vernani, de la Glossa ad Extravagantes, de la Determinatio compendiosa et de Gilles de Rome, lesquels intègrent la métaphysique de Proclus dans une argumentation voulant justifier le pouvoir universel du pape. Pour Guido Vernani, Proclus démontre la réduction de tout ordre social et politique à une communauté universelle des hommes. La Glose canoniste associe la réduction aristotélicienne à l'un dans chaque genre à la proposition 21, afin de légitimer le pouvoir universel du pape. La Determinatio compendiosa forme un chœur dans lequel Aristote, Augustin et Proclus chantent ensemble les louanges de la hiérarchie ontologique des choses, afin de réduire la causalité du mouvement à Dieu, afin d'en tirer l'origine divine du pouvoir. Gilles de Rome se réfère à la proposition $16 \mathrm{du}$ Liber de causis afin d'en déduire une théorie du monothéisme qui lui sert comme modèle politique et qui, en combinaison avec la formule (papa virarius dei), légitime la plenitudo potestatis du pape. Pierre d'Auvergne, quant à lui, utilise les propositions 21 et 5 pour justifier une théorie générale de la domination politique et sociale. Pour ceux qui en douteraient encore, ajoutons que l'amalgame du discours métaphysique et du discours politique n'est pas l'apanage de la littérature dite de propagande, mais également un phénomène présent dans les textes universitaires, tel les Questiones de Pierre d'Auvergne. Parmi nos exemples ce dernier est le plus radical: la réduction à l'un de Proclus lui apporte les moyens de légitimer une domination de type despotique dans toutes les communautés et relations humaines.

\section{La réduction à l'un: Proclus ou Aristote?}

De nombreux auteurs n'opèrent pas de distinction entre la réduction à l'un de Proclus et la réduction aristotélicienne à un premier dans chaque catégorie. Il faut une fois de plus se rapporter aux thèses de Loris Sturlese sur la réception de Proclus, afin de constater que la tendance des auteurs politiques à harmoniser les métaphysiques aristotélicienne et proclusienne est une caractéristique significative de la réception de Proclus. L'harmonisation de Proclus et d'Aristote n'est donc pas seulement le résultat de l'obsession que semblent éprouver les auteurs politiques envers la réduction à l'un.

Les textes de Vernani, de la Glose aux Extravagantes, de Pierre d'Auvergne et de la Determinatio compendiosa combinent et mélangent les éléments proclusiens à des éléments de la métaphysique aristotélicienne. 
Si nous la considérons en tant que telle, il est possible de voir chez Proclus une autre métaphysique. Mais sous l'aspect de sa réception et de sa mise en fonction politique, nous ne pouvons reconnaître qu'une même et seule métaphysique, puisque elle ne doit servir qu'à une seule politique, à savoir à la politique de la domination de l'un. Nous pouvons mieux apprécier, dans cette perspective, la critique que lançait Dante Alighieri contre une application générale de la réduction à un premier dans chaque catégorie, ainsi que le proposait Aristote dans le livre X de la Métaphysique. En appliquant la logique aristotélicienne, Dante considère que l'identification de la réduction à l'un avec la réduction de tout pouvoir au pape est une erreur de catégorie:

Ad hanc rationem solvendam dico quod, cum dicunt « Ea que sunt unius generis oportet reduci ad aliquod unum de illo genere, quod est metrum in ipso ), verum dicunt. Et similiter verum dicunt dicentes quod omnes homines sunt unius generis; et similiter verum concludunt cum inferunt ex hiis omnes homines esse reducendos ad unum metrum in suo genere. Sed cum ex hac conclusione subinferunt de Papa et Imperatore, falluntur (secundum accidens). Ad cuius evidentiam sciendum quod aliud est esse hominem et aliud est esse Papam; et eodem modo aliud est esse hominem, aliud esse Imperatorem, sicut aliud est esse hominem, et aliud est esse patrem et dominum. ${ }^{30}$

La réduction à un premier dans chaque catégorie ne peut pas consister en une réduction à un premier se constituant de manière relationnelle. La réduction des hommes aux papes est démontrée par Dante comme étant une fallacio secundum accidens, c'est-à-dire qu'elle témoigne d'une identification erronée des termes 〈homme〉 et 〈pape〉 ou 〈homme > et (père > etc. ${ }^{31}$ Nous voyons apparaître la signification de la critique dantesque quand nous constatons qu'elle prive de leur fondement théorique un grand nombre de textes politiques appliquant d'une façon générale la réduction á l'un. ${ }^{32}$ Dante illustre l'incompatibilité

30 Mon. III, xi, 3 f.: Ed. Ricci, Milan 1965, $261 \mathrm{f}$.

31 Pour une présentation de la Monarchie de Dante avec bibliographie cf. J. Miethke, «Der Weltanspruch des Papstes im späten Mittelalter», dans: I. Fetscher/H. Münkler, Pipers Handbuch der politischen Ideen, II, München 1993, 351-445. Selon Petrus Hispanus (Tractatus, VII, éd. L. M. De Rijk, 146) la fallacio secundum accidens se produit: «quando similiter quidlibet fuerit assignatum rei subiecte et accidenti inesse ".

32 Cf. aussi Bonaventure, Quaestiones disputatae de perfectione evangelica, q. 4, a. 3: De oboedientia summi Pontifici debita, in: Doctoris seraphici Opera omnia, studio et cura PP. Collegii a S. Bonaventura, t. V, Quaracchi 1891, 193b-194b: "Naturalis namque iustitia, per quam ordinantur elementa mundi et tota machina universi, requirit, quod inter corpora locantia unum sit primum corpus locans, inter mobilia unum primum mobile, inter lucentia unum principaliter 
de la réduction à un premier homme avec l'application générale de la réduction à l'un à toutes les relations humaines. Bon nombre des textes politiques présentés ici ont omis de faire une distinction entre Aristote et Proclus, en ce qui a trait à la réduction à l'un. Dans ce sens, la critique de Dante touche aussi l'application politique de la réduction proclusienne.

\section{La réduction à l'un comme principe politique}

A la lumière des textes que nous avons examinés, il est permis de conclure que le métaphysicien Proclus a aussi joué un rôle politique. Tous les auteurs cités suivent la méthode d'une intégration de la métaphysique dans la politique, d'une mise en service de principes métaphysiques pour étayer des thèses politiques. Il leur aurait été difficile de résister à la tentation de faire usage, dans leur argumentation politique, de la pertinente formulation de la réduction à l'un offerte par Proclus. (Omnis multitudo ad unam reducitur unitatem) est ainsi devenu une formule qui a servi comme principe universel dans des syllogismes pratiques, et s'est vu doté d'une fonction axiomatique dans un procédé de légitimation générale soit de la domination despotique tout court, soit du pouvoir papal. Compte tenu de ce qui précède l'histoire de la réception de Proclus au moyen âge doit alors concéder une place relativement importante au Proclus politisé. Une bonne part des éléments politiques légitimisés par la pensée de Proclus correspondaient aux réalités politiques du moyen âge. A l'exception de quelques ordres religieux et de quelques villes, monarchie et hiérarchie sont des structures omniprésentes dans la société médiévale. On voit donc qu'une partie de la spéculation proclusienne a au moyen âge rempli une fonction de légitimation politique. Face à cette mise en fonction politique de la métaphysique, aristotélicienne ou néoplatonicienne, je risque l'hypothèse

irradians, inter motores unus primus motor, et universaliter « in quolibet genere sit unum primum, per quod mensurentur omnia, quae in illo genere continentur ». [...] Quoniam igitur haec inferior hierarchia et ordini naturali consonat et morali et caelesti, quia naturam perficit et mores ornat et a superna Ierusalem emanat; hinc est, quod ipsa reduci habet secundum rationem obedientiae ad unum primum et summum. Sicut enim non est ordo prioritatis et posterioritatis nisi per reductionem ad primum, sic non est ordo completus secundum sub et supra nisi per reductionem ad summum. [...] Ubi vero est reductio ad summum in genere hominum, cuiusmodi est Christi Vicarius, Pontifex summus; ibi est ordo perfectus. 》 
qu'on ne peut comprendre la signification historique d'une théorie métaphysique qu'en tenant compte de sa possible ou actuelle mise en fonction politique. Le prétendu, ou véritable, caractère immobile, éternel, universel et même divin de la métaphysique se prête d'une façon idéale au discours de légitimation politique et morale. Le discours métaphysique, comme le disait Kant, semble indispensable si on veut atteindre une philosophie pratique de caractère universel. Pour une époque de l'histoire de la philosophie qui comprenait l'ordre politique comme imitation de l'ordre cosmologique et ontologique, et ce présupposé est à la base de toutes les argumentations évoquées ici, les doctrines métaphysiques et cosmologiques sont, par nécessité, des doctrines aux implications politiques. Soit dans un discours ecclésiologique, soit dans un discours sur le pouvoir papal, soit dans un discours philosophique sur la théorie générale de la domination, la réduction à l'un de Proclus légitime ainsi la domination de l'un. "Denken des Einen " signifie toujours, dans ce cas, "Herrschen des Einen ". La réduction à l'un comme doctrine métaphysique assure à la réduction à l'un politique, les arguments nécessaires à l'établissement des assises philosophiques de sa domination. Dans cette perspective fonctionnaliste, mais aussi dans les textes eux-mêmes, la métaphysique de l'un est ainsi une métaphysique despotique. 